\title{
Recent Results On Heavy Flavor Production At RHIC-PHENIX
}

Takashi Hachiya* for the PHENIX collaboration

RIKEN BNL Research Center

E-mail: hachiya@rcf.rhic.bnl.gov

\begin{abstract}
Bottom and charm productions has been measured separately in $\mathrm{Au}+\mathrm{Au}$ collisions at mid rapidity and $\mathrm{Cu}+\mathrm{Au}$ collisions at forward rapidity at $\sqrt{s_{N N}}=200 \mathrm{GeV}$ using the PHENIX silicon tracking system. In $\mathrm{Au}+\mathrm{Au}$ collisions, we measured single electrons from bottom and charm hadron decays separately and found that bottoms are less suppressed than charms at $3<p_{T}<4 \mathrm{GeV} / \mathrm{c}$. In $\mathrm{Cu}+\mathrm{Au}$ collisions, we measured $J / \psi$ from $B$ meson decay and found that the $B$ meson production at low $p_{T}$ is consistent with number of binary collision scaling at forward rapidity.
\end{abstract}

The 26th International Nuclear Physics Conference

11-16 September, 2016

Adelaide, Australia

\footnotetext{
* Speaker.
} 


\section{Introduction}

Hadrons carrying heavy quarks, bottoms and charms, are a powerful probe to study the property of quark gluon plasma (QGP) created in high energy heavy ion collisions. Heavy quarks are mainly produced via initial hard scattering at the early stage of collision because of their large masses $\left(M_{b} \approx 4.2 \mathrm{GeV} / c^{2}\right.$ and $\left.M_{c} \approx 1.3 \mathrm{GeV} / c^{2}\right)$. Once produced, number of heavy quarks are preserved when they traverse QGP. However they suffer the final state effects such as energy loss and collective flow in QGP. Consequently, the momentum and angular distribution of heavy quarks can be modified.

PHENIX studied a variety of heavy quark productions in $p+p$ and Au+Au collisions. We previously measured a strong suppression and a substantial elliptic flow of heavy quarks in the measurement of single electrons from semi-leptonic decays of hadrons containing bottom and charm quarks[1,2]. The results were striking since it was expected that heavy quarks lose less energy due to a suppression of gluon radiation such as the dead cone effect[3]. To investigate the heavy quark suppression in detail, it is necessary to separate the bottom and charm contributions. For the specific purpose, a Barrel and Forward Silicon Vertex Tracker (VTX and FVTX) were installed in PHENIX in 2011 and 2012. VTX (FVTX) covers $|y|<1.2(1.2<|y|<2.2)$ in rapidity and almost $2 \pi$ (full) in azimuth. These silicon detectors provide precise displaced trackings which enables the separation of bottom and charm components by measuring their decay lengths. In addition, $J / \psi$ from $B$ decay is a clean channel to measure bottom yield. In this proceedings, we report on separated bottom and charm yields at mid rapidity in $\mathrm{Au}+\mathrm{Au}$ collisions and $J / \psi$ from $B$ decay at forward rapidity in $\mathrm{Cu}+\mathrm{Au}$ collisions.

\section{Separated bottom and charm yields in $\mathrm{Au}+$ Au collisions}

This analysis is performed using data from $\mathrm{Au}+\mathrm{Au}$ collisions at $\sqrt{s_{N N}}=200 \mathrm{GeV}$ in 2011. We separate the electrons from bottom and charm hadron decays using the difference of their decay lengths, where the decay length of $B$ meson is longer than that of $D$ mesons $\left(c \tau_{B^{0}} \approx 455 \mu \mathrm{m}\right.$ and $c \tau_{D^{0}} \approx 123 \mu \mathrm{m}$ ). Electrons, first, are identified using a ring-imaging Čerenkov detector and the energy-momentum matching. VTX measures the distance of closest approach (DCA) of the track to the collision vertex. The DCA is broader for electrons from bottom decays than that from charm decays. Inclusive electrons contains a large amount of backgrounds that originate from non-desired physics processes such as Dalitz decays of $\pi^{0}$ and $\eta$, and photon conversions as well as detector effects such as mis-identified hadrons and high multiplicity background. Those backgrounds in the DCA distribution are estimated and subtracted using the event driven method and the PHENIX detector simulations. The remaining signal electrons in the DCA distribution are statistically separated to the bottom and charm components by the unfolding method based on Bayesian inference technique. The unfolding method performs a simultaneous fitting to both the DCA distributions and the invariant yield of electrons from inclusive heavy quark decays. The analysis procedure is described in detail[4]. Figure 1 shows the comparisons between measured electrons and calculations by the unfolding method in $p_{T}$ (left) and DCA distribution (right), respectively. The bottom panels are their ratios. From these ratios, they are in good agreement in both invariant yield and 


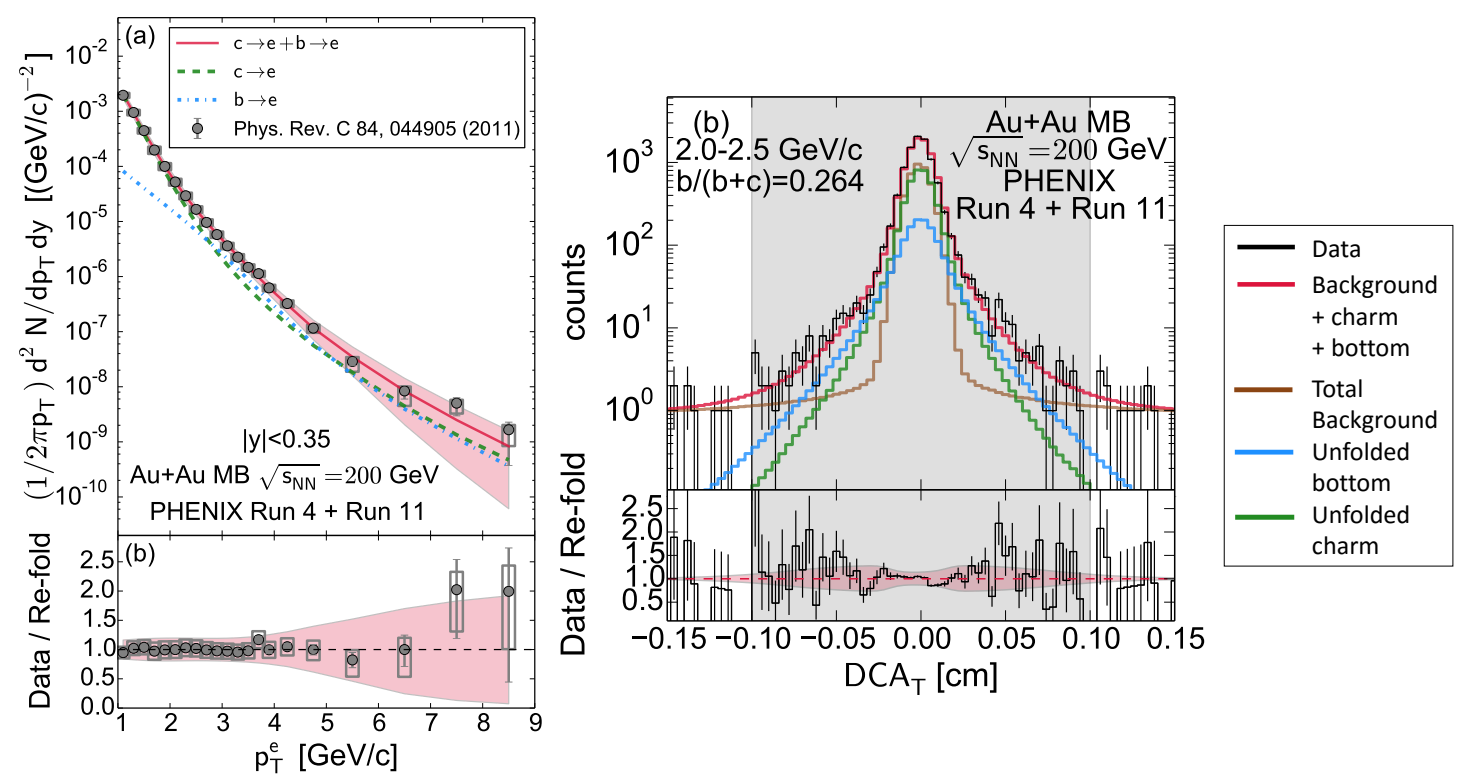

Figure 1: (Left) Invariant yield of inclusive electron from heavy quark decays [2]. (Right) DCA distribution for electrons measured in this analysis. These distributions are compared with the calculated bottom and charm components from the unfolding method as well as their backgrounds. Bottom panels show the ratio between the data and the calculation from the unfolding method.

DCA within the uncertainties. This indicates that the simultaneous fitting by the unfolding method works well.

Figure. 2 (left) shows a fraction of electrons from bottom decays to that from inclusive heavy quarks. The red curve and pink band represent the central value and systematic uncertainties. The resulting bottom electron fraction shows a possible peak at $p_{T} \sim 3 \mathrm{GeV} / c$. The bottom electron fraction is compared with the FONLL predictions[5] and the $p+p$ results[6, 7]. Here, these $p+p$ results increase smoothly. We found that our $\mathrm{Au}+\mathrm{Au}$ result is consistent with $p+p$ results for $p_{T}>$ $4 \mathrm{GeV} / \mathrm{c}$ within uncertainty, and the shape of the bottom electron fraction at low $p_{T}$ is apparently different. The large uncertainty of FONLL prediction covers both $\mathrm{Au}+\mathrm{Au}$ and $p+p$.

The nuclear modification factor $\left(R_{A A}\right)$ for electrons from bottom and charm hadron decays are calculated using the bottom electron fraction in $\mathrm{Au}+\mathrm{Au}$ and $p+p$, and $R_{A A}$ of electrons from inclusive heavy quark decays[2]. These $R_{A A}$ are plotted in the right panel of Fig. 2. We found that electrons from bottom decays are less suppressed than that from charm decays for $p_{T}<4 \mathrm{GeV} / c$ and similarly suppressed for higher $p_{T}$. This result is qualitatively consistent with the expectations from the mass dependence of energy loss[3, 8].

\section{Open bottom yield via $J / \psi$ from $B$ meson decays in $\mathrm{Cu}+$ Au collisions}

The analysis was performed using data from $\sqrt{s_{N N}}=200 \mathrm{GeV} p+p$ collisions in 2015 and $\mathrm{Cu}+\mathrm{Au}$ collisions in 2012. $J / \psi$ from $B$ meson decays is a clean measurement of open bottom production. PHENIX measure the signal via $B \rightarrow J / \psi(+X) \rightarrow \mu^{+}+\mu^{-}$at forward and backward rapidity. $J / \psi$ is reconstructed from unlike sign di-muon pairs within the invariant mass $2.8<M_{\mu \mu}$ $\left(\mathrm{GeV} / c^{2}\right)<3.5$. FVTX measures DCA of single muons from $J / \psi$ decays. $J / \psi$ from $B$ decay 

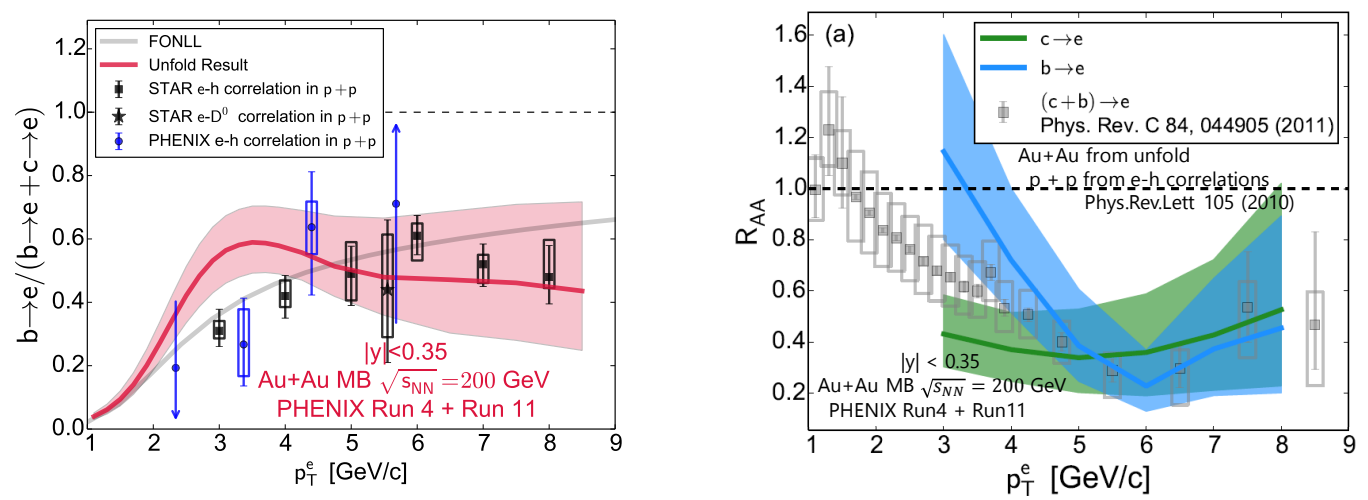

Figure 2: (Left) The bottom electron fraction in minimum bias Au + Au collisions at $\sqrt{s_{N N}}=200 \mathrm{GeV}$ [4] the FONLL prediction[5] and the $p+p$ results from PHENIX[6] and STAR[7] are plotted for comparison. (Right) $R_{A A}$ for electrons from separated bottom and charm hadron decays as well as inclusive heavy flavor electrons.

originate far from the collision vertex. The DCA distribution for $J / \psi$ from $B$ is broader than that of prompt $J / \psi$. In addition, the DCA distribution for the $B$ decays has longer tail in negative side because the negative side has better detector acceptance, while prompt $J / \psi$ from the collision vertex is symmetric. The difference of their shapes in the DCA distribution is used to separate muons from $J / \psi$ from $B$ decays and prompt $J / \psi$. The DCA distribution is fit with the template DCA shapes of $J / \psi$ from B decays and prompt $J / \psi$ to extract the $B$ decay components. Their template shapes are determined by PYTHIA and PHENIX detector simulation. The analysis procedure is described in detail[9]. Figure 3 shows the DCA distribution of muons from $J / \psi$ decays in $p+p$ collisions. The fit results are also plotted for comparison. In the fit procedure, several energy loss scenarios which can modify the template shapes are tested. The resulting differences from these scenarios are taken as systematic uncertainty.

Figure 4 shows the fraction of $J / \psi$ from $B$ decays relative to inclusive $J / \psi$ as a function of rapidity in $200 \mathrm{GeV} p+p$ and $\mathrm{Cu}+\mathrm{Au}$ collisions. The results are compared with the FONLL prediction[10] for $J / \psi$ from $B$ decay and Color Evaporation Model[11] for prompt $J / \psi$. The fraction in $p+p$ is found to be well described by the prediction. On the other hand, the fractions for both $\mathrm{Cu}$ - and $\mathrm{Au}$ - going direction are systematically larger than that in $p+p$. This indicates that B meson is less suppressed than prompt $J / \psi$.

The nuclear modification factor $R_{A A}$ of $J / \psi$ from B decays and prompt $J / \psi$ is calculated using the fractions in $\mathrm{Cu}+\mathrm{Au}$ and $p+p$, and the $R_{A A}$ of inclusive $J / \psi[12]$. Figure 5 shows these $R_{A A}$ as a function of rapidity. The $R_{A A}$ of $J / \psi$ of B decay favors an slight enhancement at Au going direction (negative rapidity), even though they are consistent with unity within uncertainties for both $\mathrm{Cu}$ - and $\mathrm{Au}$ - going directions. This indicates that $\mathrm{B}$ meson production is scaled with number of binary nucleon-nucleon collisions.

\section{Summary}

PHENIX measured heavy quark productions in $\mathrm{Au}+\mathrm{Au}$ and $\mathrm{Cu}+\mathrm{Au}$ collisions at $\sqrt{s_{N N}}=200$ GeV. VTX and FVTX provided new capability to separate the bottom and charm contributions in 


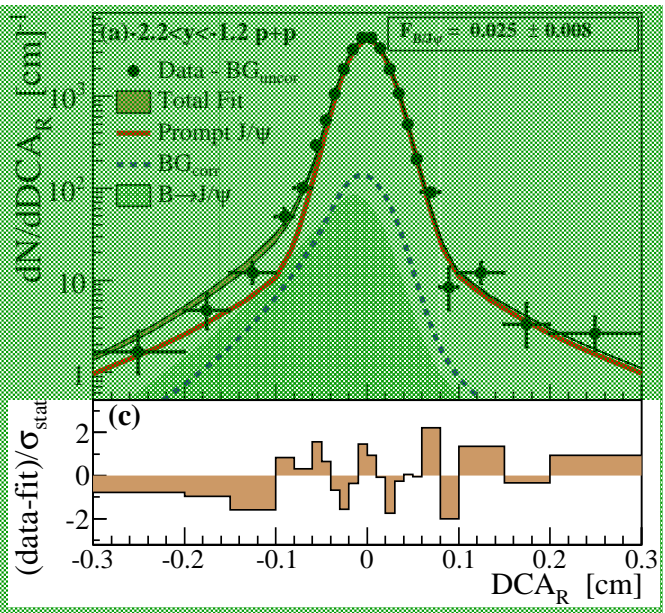

Figure 3: DCA distribution of single muon from $J / \psi$ decays in $p+p$ collisions at $\sqrt{s_{N N}}=$ $200 \mathrm{GeV}$. The fit functions for $J / \psi$ from $B$ decay and prompt $J / \psi$, and the background component are plotted.

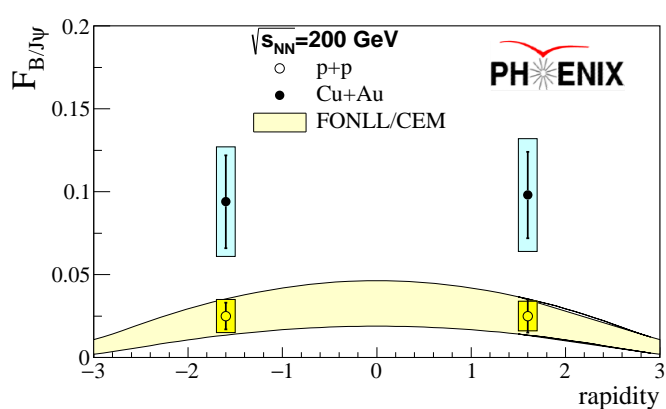

Figure 4: The fraction of $J / \psi$ from $B$ decays relative to inclusive $J / \psi$ as a function of rapidity in $\mathrm{Cu}+\mathrm{Au}$ and $p+p$ collisions at $\sqrt{s_{N N}}=200$ $\mathrm{GeV}$

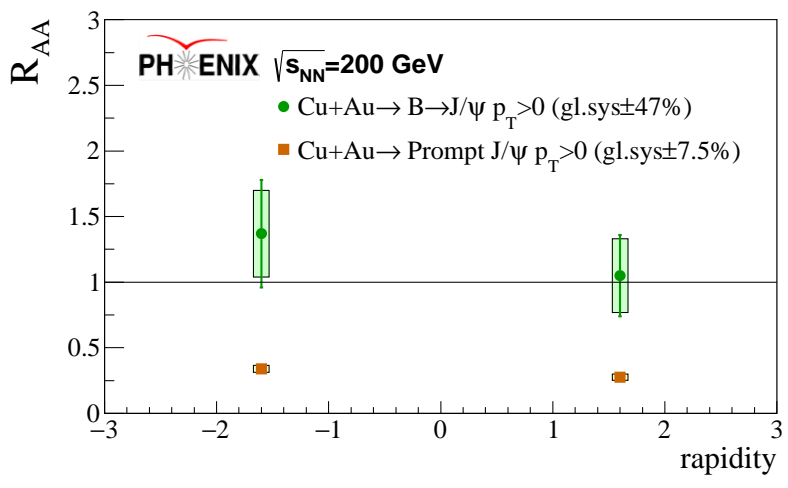

Figure 5: $R_{A A}$ of $J / \psi$ of $B$ decays and prompt $J / \psi$ as a function of rapidity in $\mathrm{Cu}+\mathrm{Au}$ collisions at $\sqrt{s_{N N}}$ $=200 \mathrm{GeV}$.

the single electron measurement in $\mathrm{Au}+\mathrm{Au}$ collisions and to identify $J / \psi$ from $B$ decays in $\mathrm{Cu}+\mathrm{Au}$ collisions. For the first time, we found the less suppression of electrons from bottom decays than that from charm decays for $3<p_{T}<4 \mathrm{GeV} / \mathrm{c}$ and similar suppression for higher $p_{T}$ at RHIC energy. The first result of open bottom production in $\mathrm{Cu}+\mathrm{Au}$ collisions shows no suppression within uncertainty for both $\mathrm{Cu}$ - and $\mathrm{Au}$ - going direction, which is consistent with number of binary collision scaling. These results on the heavy quark production look into the QGP property from new angle. We note that significantly large amount of $\mathrm{Au}+\mathrm{Au}$ and $p+p$ data were collected in 2014 to 2016. These new dataset provide both an important baseline measurement in $p+p$ and a more precise measurement in $\mathrm{Au}+\mathrm{Au}$ collisions. 


\section{References}

[1] A. Adare et al. Energy Loss and Flow of Heavy Quarks in Au $+\mathrm{Au}$ Collisions at $\sqrt{s_{N N}}=200 \mathrm{GeV}$ Phys. Rev. Lett. 98172301 (2007)

[2] A. Adare et al. Heavy-quark production in $p+p$ and energy loss and flow of heavy quarks in $\mathrm{Au}+\mathrm{Au}$ collisions at $\sqrt{s_{N N}}=200 \mathrm{GeV}$ Phys. Rev. C 84, 044905 (2011)

[3] Y. L. Dokshitzer and D. E. Kharzeev Phys. Lett. B 519, 199 (2001)

[4] A. Adare et al. Single electron yields from semileptonic charm and bottom hadron decays in $\mathrm{Au}+\mathrm{Au}$ collisions at $\sqrt{s_{N N}}=200 \mathrm{GeV}$ Phys. Rev. C 93034904 (2016)

[5] M. Cacciari, P. Nason, and R.Vogt, QCD Predictions for Charm and Bottom Quark Production at RHIC Phys. Rev. Lett. 95122001 (2005)

[6] A. Adare et al. Measurement of Bottom versus Charm as a Function of Transverse Momentum with Electron-Hadron Correlations in $p+p$ Collisions at $\sqrt{s}=200 \mathrm{GeV}$ Phys. Rev. Lett. 103082002 (2009)

[7] M. Aggarwal et al. Measurement of the Bottom contribution to non-photonic electron production in $p+p$ collisions at $\sqrt{s}=200 \mathrm{GeV}$ Phys. Rev. Lett. 105202301 (2010)

[8] A. Adil and I. Vitev Collisional dissociation of heavy mesons in dense QCD matter Phys. Lett. B 649 139-146 (2007)

[9] C. Aidala et al., B-meson nuclear modification at forward and backward rapidity in $\mathrm{Cu}+\mathrm{Au}$ collisions at $\sqrt{s_{N N}}=200 \mathrm{GeV}$, arXiv:1702.01085

[10] M. Cacciari, M. Greco, and P. Nason, The $p_{T}$ Spectrum in Heavy-Flavour Hadroproduction, JHEP 05, 007 (1998)

[11] A. D. Frawley, T. Ullrich, and R. Vogt, Heavy flavor in heavy-ion collisions at RHIC and RHIC II, Phys. Rept. 462,790 125 (2008)

[12] C. Aidala et al., Nuclear matter effects on $J / \psi$ production in asymmetric $\mathrm{Cu}+\mathrm{Au}$ collisions at $\sqrt{s_{N N}}=$ 200 GeV, Phys. Rev. C 90064908 (2014) 